\title{
Research on Vehicle Scheduling Optimization Problem of Dangerous Goods Transportation under Single Distribution Center based on Security
}

\author{
Kun $\mathrm{Zou}^{1}$, Jin $\mathrm{Du}^{1}$ \\ ${ }^{1}$ Yinxing Hospitality management College, Chengdu University of Information Technology, Chengdu, \\ 611743, China
}

Keywords: Security, dangerous goods, vehicle scheduling optimization, transportation

\begin{abstract}
Since the 21st century, with the continuous economic growth, the demand for dangerous chemicals has been growing and its safe transportation problem has drawn close attention of the society and government. This paper systematically elaborates the research status of vehicle scheduling optimization problem at home and abroad, establishes a vehicle scheduling optimization model of dangerous chemicals transportation under single distribution center based on security, designs a genetic algorithm to solve the model, and finally verifies the reliability of the model and validity of the algorithm by the design example.
\end{abstract}

\section{Introduction}

Through the continuous efforts of many scholars, the research on vehicle scheduling problem in China has caused the attention of the relevant departments, and also made some preliminary results over the years. In all areas of economic development, the vehicle transportation problem with the time window is very important and has a certain realistic significance. The vehicle optimization problem of non-full load vehicle is analyzed with the relevant theory of by the related theory of mathematical programming; meanwhile, the fixed time window, travel distance, maximum bearing capacity of transport vehicle and other constraints are analyzed and studied, which are of great significance in saving the operation cost, maximizing the economic benefits, and realizing the effective conversion of enterprise economic profits.

\section{Problem description and assumption}

\section{Problem description}

For the transportation of dangerous chemical under single distribution center, the main objectives of study and solution are:

(1) Ensure the efficiency of distribution. It is required to send the goods required by a customer to the designated location on time in accordance with the requirements of the customer to improve the service quality and accelerate the efficiency of logistics distribution.

(2) How to design a reasonable route to minimize the number of total transport vehicle, total travel distance of transport vehicle, number of densely populated areas and restricted areas that the vehicle goes through. It is best to use this multi-target to reflect the safety of road vehicle transportation of dangerous chemicals, which is the main objective of this system.

Model assumption

Based on the above description, the following assumption are made: (1) There is one dangerous chemical distribution center in the whole distribution system. (2) The locations of distribution center location and customer are known in advance and unchanged. (3) Customers' demand for dangerous chemicals or the supply are known in advance and unchanged. (4) The carrying capacity of each vehicle in the transportation of dangerous chemicals transportation is less than its capacity. (5) The demand of each customer for dangerous chemicals is not more than the maximum capacity of each vehicle. (6) The time window of each customer is known. (7) Whether the customers go through the densely populated areas is known in advance. (8) Whether the customers go through the restricted 
zones is known in advance. (9) The demands of every customer are met only with a vehicle. (10) There is one transportation route at most between two demand points.

\section{Model building}

\section{Symbol definition}

Parameters:

$C$ : Set of customers requiring service, $i=1,2, \cdots,|C|$;

$\theta$ : Distribution center, $i=0$;

$N$ : Node set, $N=\{\theta\} \cup C$;

$K$ : Set of dangerous goods transport vehicles, $k=1,2, \cdots,|K|$;

CAP : Capacity of distribution vehicle;

$K_{c}$ : Fixed cost of dangerous goods transport vehicles;

$q_{i}$ : Demand of customer $i, i \in C$;

$\left[E T_{i}, L T_{i}\right]$ : Time window of customer $i$, where $E T_{i}$ is the earliest arrival time of goods and $L T_{i}$ is the latest arrival time of goods;

$d_{i j}$ : Travel distance from node $i$ to node $j$;

$c_{i j k}$ : Transportation cost of unit distance of vehicle $k$ from node $i$ to node $j$;

$t_{i j}$ : Time from node $i$ to node $j$;

$T_{i k}$ : Arrival time of vehicle $k$ at node $i$;

$P_{(i, j)}$ : Set of forbidden zones from node $i$ to node $j$;

$G_{(i, j)}^{l}$ : Cost of distribution vehicle going through forbidden zones $l$ between node $i$ and node $j$;

$V_{(i, j)}$ : Set of schools, villages, towns and military administrative zones and other densely populated areas from node $i$ to node $j$;

Decision variable:

$x_{i j k}$ : if vehicle $k$ arrives at node $j$ through node $i, x_{i j k}=1$ and $x_{i j k}=0$;

$p_{i j k}^{l}$ : if vehicle $k$ arrives at forbidden zone $l$ in node $j$ through node $i, p_{i j k}^{l}=1$, and $p_{i j k}^{l}=0$;

$v_{i j k}^{l}$ : if vehicle $k$ arrives at densely populated area $l$ in node $j$ through node $i, v_{i j k}^{l}=1$, and $v_{i j k}^{l}=0$;

\section{Building of mathematical model}

$$
\begin{gathered}
Z_{1}=\min \underbrace{\sum_{i \in C} \sum_{j \in C} \sum_{k \in K} d_{i j} \cdot c_{i j k} \cdot x_{i j k}}_{1}+\underbrace{\sum_{j \in C} \sum_{k \in K} K_{c} \cdot x_{0 j k}}_{2}+\underbrace{\sum_{i \in N} \sum_{j \in N} \sum_{k \in K} \sum_{l=1}^{\left|P_{i, j}\right|} G_{(i, j)}^{l} \cdot p_{i j k}^{l} \cdot x_{i j k}}_{3} \\
Z_{2}=\min \sum_{i \in N} \sum_{j \in N} \sum_{k \in K} \sum_{l=1}^{\left|V_{(i, j)}\right|} v_{i j k}^{l} \cdot x_{i j k} \\
\text { s.t. } \sum_{i \in N} \sum_{k \in K} x_{i j k}=1, \quad \forall j \in C \\
\sum_{j \in C} q_{j} \cdot \sum_{i \in C} x_{i j k} \leq C A P, \quad \forall k \in K \\
\sum_{j \in N} x_{i j k}-\sum_{j \in N} x_{j i k}=0, \quad \forall i \in N, \quad \forall k \in K \\
E T_{i} \leq T_{i k} \leq L T_{i}, T_{i k}=T_{j k}+t_{i j} \cdot x_{i j k}, \quad \forall i \in C, \quad \forall k \in K \\
x_{i j k}=\{0,1\}, \quad \forall i, j \in N, \quad \forall k \in K \\
p_{i j k}^{l}=\{0,1\}, \quad \forall i, j \in N, \quad \forall k \in K, \quad \forall l \in P_{(i, j)} \\
v_{i j k}^{l}=\{0,1\}, \quad \forall i, j \in N, \quad \forall k \in K, \quad \forall l \in V_{(i, j)}
\end{gathered}
$$

Where, the target equation (1) refers to the minimum distribution cost, including three parts: part 
one is the transportation cost, part two is the fixed costs of vehicle, and part three is the cost of distribution vehicles passing through the forbidden areas. Target equation (2) refers to the minimum number of densely populated concentration areas, that is, the possibility of risk is the least . Constraint equation (3) means that there is only one vehicle serving each customer. Constraint equation (4) means the demand of customers served by vehicle $k$ is not less than the carrying capacity of vehicle. Constraint equation (5) means the vehicle at the point must move away from that point, and refers to the distribution center when $j=0$, that is, the vehicle starting from the distribution center must return to distribution center. Constraint equation (6) refers to the constraints of time window.Constraint equations (7), (8) and (9) refer to 0-1 variable constraint.

\section{Model solution}

\section{Processing of time window}

For constraint equation (6), this paper punishes the distribution scheme that fails to meet the time window requirements wit the punitive measures and then form the total cost of this distribution scheme with objective function 1. It is known from equation (3-6) that $T_{i k}=T_{j k}+t_{i j} \cdot X_{i j k}$, when $T_{i k}<E T_{i}$, the penalty value $P F_{1}=Q_{1} \cdot\left(E T_{i}-T_{i k}\right)$; when $T_{i k}>L T_{i}$, the penalty value $P F_{2}=Q_{2} \cdot\left(T_{i k}-L T_{i}\right)$, where $Q_{1}$ and $Q_{2}$ are the penalty coefficients. Then model $Z_{1}$ will be:

$$
\begin{aligned}
& Z_{3}=\min \underbrace{\sum_{i \in C} \sum_{j \in C} \sum_{k \in K} d_{i j} \cdot c_{i j k} \cdot x_{i j k}}_{1}+\underbrace{\sum_{j \in C} \sum_{k \in K} K_{c} \cdot x_{o j k}}_{2}+\underbrace{\sum_{i \in C} \sum_{j \in C} \sum_{k \in K} \sum_{l=1}^{\left|P_{i, j, j}\right|} G_{(i, j)}^{l} \cdot p_{i j k}^{l} \cdot x_{i j k}}_{3} \\
& +\underbrace{F_{1} \cdot P F_{1}+\left(1-F_{1}\right) \cdot P F_{2}}_{4}
\end{aligned}
$$

Where, item 4 in equation (14) refers to the penalty value of time window and $F_{1}=\left\{\begin{array}{ll}1 & T_{i k}<E T_{i} \\ 0 & T_{i k}>L T_{i}\end{array}\right.$.

\section{Multi-target processing}

After dimension of the value of target equation (3-2) and (3-10), the weight $\omega_{2}$ and $\omega_{3}$ are given respectively, and $0 \leq \omega_{2} \leq 1,0 \leq \omega_{3} \leq 1$ and $\omega_{2}+\omega_{3}=1$. The values of $\omega_{2}$ and $\omega_{3}$ are confirmed through the actual research of dangerous goods transportation industry.

Suppose that the minimum value is $Z_{2}^{\max }$ and the minimum value is $Z_{2}^{\min }$ in target equation (2) and the minimum value is $Z_{3}^{\max }$ and the minimum value is $Z_{3}^{\min }$ in target equation (3), for target equation (2) and (10), the value after the unified dimension of current value can be dimensioned by the following equation.

$$
Z_{i}^{\prime}=\frac{Z_{i}-Z_{i}^{\min }}{Z_{i}^{\max }-Z_{i}^{\min }}, \quad i=2,3
$$

Thus, the comprehensive target value can be expressed as $Z=\min \sum_{i} \omega_{i} \cdot z_{i}^{\prime}$, and $i=2,3$.

\section{Genetic algorithm}

\section{(1) Chromosome coding}

The problem to be solved in this chapter is the route arrangement of distribution center. Thus, the corresponding genetic algorithm coding adopts the natural number coding. The chromosome coding confirms the customer service sequence of distribution center and distribute the vehicle according to its capacity. The vehicle length is $|C|$ and gene bit is a set of random arrangement before $[1,|C|]$.

\section{(2) Initial population and fitness function}

Suppose that the chromosome population size is Popsize and the initial population is formed by generating a chromosome randomly. Then, the fitness of initial population is calculated. In this paper, the fitness function $f_{l}=1 / z_{l}$, where $Z_{l}$ is the target value of chromosome $l$ in population. 


\section{(3) Crossover and mutation operation}

To prevent error code in the crossover and mutation process, the ordered crossover and partial matched crossover as well as reverse transcription variation are adopted.

\section{(4) Selecting operation}

In this paper, the chromosome required by crossover and mutation is selected by roulette, the selected chromosome is copied as the parent chromosome, then crossover and mutation are carried out, the fitness value of newly formed daughter chromosomes is calculated, which is recombined with the original chromosome, and finally the chromosome with great fitness of Popsize selected by elitism selection strategy enters the next generation. After selecting operation, the crossover, mutation and recombination are conducted to form a new population.

\section{(5) Algorithm stop condition}

When the iterative algebra of genetic algorithm is maximized, it is required to stop iteration and output the results.

\section{Analysis of examples}

\section{Structure of examples}

There is an oil distribution center, with a storage capacity of 150 tons of oils, position coordinates of $(18,15), 30$ self-owned oil tank truckers, the maximum carrying capacity of which is $C A P=20$ tons. The distribution vehicles must return to the distribution center after completing the distribution task and no longer undertake any other distribution tasks. Suppose that the fixed cost of a new vehicle $K_{c}=800$ yuan. The oil distribution center should serve 24 demand points, its geographical location information is shown in table 1, the distance and operation time between two points are shown in literature [7], the number of densely populated areas between two points is shown in table 2, and the number of forbidden zones between two points and the cost of going through the forbidden zones are shown in table 3.

Table 1. Customer information and data sheet

\begin{tabular}{|c|c|c|c|c|c|c|c|c|c|c|c|}
\hline \multirow{2}{*}{ Serial No. } & \multicolumn{2}{|c|}{ Coordinate } & \multirow{2}{*}{ Demand } & \multirow{2}{*}{$E T_{i}$} & \multirow{2}{*}{$L T_{i}$} & \multirow{2}{*}{ Serial No. } & \multicolumn{2}{|c|}{ Coordinate } & \multirow{2}{*}{ Demand } & \multirow{2}{*}{$E T_{i}$} & \multirow{2}{*}{$L T_{i}$} \\
\hline & $\mathrm{X}$ & $\mathrm{Y}$ & & & & & $\mathrm{X}$ & $\mathrm{Y}$ & & & \\
\hline 1 & 20 & 85 & 4.2 & 0 & 14 & 11 & 25 & 30 & 4 & 0 & 16 \\
\hline 2 & 5 & 45 & 7.2 & 0 & 8 & 12 & 15 & 10 & 3 & 0 & 23 \\
\hline 3 & 42 & 15 & 2.5 & 0 & 11 & 13 & 45 & 65 & 3.3 & 0 & 16 \\
\hline 4 & 38 & 5 & 5.6 & 0 & 19 & 14 & 65 & 20 & 5.8 & 0 & 7 \\
\hline 5 & 95 & 35 & 7.9 & 0 & 22 & 15 & 31 & 52 & 7.9 & 0 & 11 \\
\hline 6 & 85 & 25 & 5 & 0 & 8 & 16 & 2 & 60 & 4.9 & 0 & 13 \\
\hline 7 & 62 & 80 & 2 & 0 & 14 & 17 & 5 & 5 & 3.7 & 0 & 18 \\
\hline 8 & 58 & 75 & 3.1 & 0 & 20 & 18 & 57 & 29 & 5.3 & 0 & 16 \\
\hline 9 & 50 & 50 & 4.1 & 0 & 17 & 19 & 4 & 18 & 4.9 & 0 & 18 \\
\hline 10 & 18 & 80 & 6 & 0 & 9 & 20 & 26 & 35 & 5 & 0 & 22 \\
\hline
\end{tabular}

Table 2. Number of densely populated areas between two nodes

\begin{tabular}{c|c|c|c|c|c|c|c|c|c|c|c|c|c|c|c|c|c|c|c|c|c|c|c|c|c}
\hline & 0 & 1 & 2 & 3 & 4 & 5 & 6 & 7 & 8 & 9 & 10 & 11 & 12 & 13 & 14 & 15 & 16 & 17 & 18 & 19 & 20 & 21 & 22 & 23 & 24 \\
\hline 0 & 0 & 4 & 3 & 0 & 0 & 0 & 1 & 2 & 0 & 5 & 2 & 5 & 0 & 2 & 1 & 2 & 5 & 0 & 1 & 5 & 2 & 3 & 0 & 2 & 1 \\
\hline 1 & 0 & 0 & 1 & 1 & 1 & 5 & 2 & 4 & 0 & 3 & 4 & 3 & 3 & 1 & 0 & 5 & 3 & 3 & 5 & 3 & 0 & 3 & 2 & 5 & 1 \\
\hline 2 & 0 & 0 & 0 & 5 & 0 & 0 & 0 & 5 & 3 & 0 & 3 & 5 & 5 & 0 & 4 & 3 & 3 & 2 & 1 & 2 & 3 & 4 & 4 & 0 & 2 \\
\hline 3 & 0 & 0 & 0 & 0 & 2 & 1 & 5 & 0 & 3 & 3 & 0 & 4 & 4 & 2 & 3 & 5 & 1 & 5 & 2 & 3 & 5 & 3 & 4 & 2 & 5 \\
\hline 4 & 0 & 0 & 0 & 0 & 0 & 3 & 1 & 3 & 4 & 1 & 4 & 2 & 5 & 1 & 4 & 1 & 2 & 0 & 4 & 1 & 3 & 0 & 2 & 1 & 1 \\
\hline 5 & 0 & 0 & 0 & 0 & 0 & 0 & 3 & 1 & 1 & 4 & 5 & 1 & 2 & 2 & 3 & 3 & 4 & 0 & 1 & 4 & 5 & 1 & 5 & 5 & 4 \\
\hline 6 & 0 & 0 & 0 & 0 & 0 & 0 & 0 & 4 & 3 & 2 & 1 & 2 & 3 & 0 & 0 & 1 & 5 & 1 & 1 & 4 & 5 & 0 & 5 & 0 & 1 \\
\hline 7 & 0 & 0 & 0 & 0 & 0 & 0 & 0 & 0 & 4 & 4 & 1 & 1 & 0 & 5 & 2 & 4 & 3 & 5 & 2 & 4 & 0 & 0 & 5 & 3 & 1 \\
\hline 8 & 0 & 0 & 0 & 0 & 0 & 0 & 0 & 0 & 0 & 5 & 3 & 2 & 4 & 4 & 4 & 1 & 0 & 5 & 3 & 2 & 1 & 4 & 0 & 5 & 1 \\
\hline 9 & 0 & 0 & 0 & 0 & 0 & 0 & 0 & 0 & 0 & 0 & 4 & 2 & 1 & 1 & 4 & 0 & 1 & 4 & 5 & 5 & 5 & 2 & 3 & 5 & 0 \\
\hline 10 & 0 & 0 & 0 & 0 & 0 & 0 & 0 & 0 & 0 & 0 & 0 & 1 & 2 & 3 & 4 & 5 & 1 & 3 & 3 & 3 & 1 & 0 & 4 & 0 & 3 \\
\hline
\end{tabular}




\begin{tabular}{l|l|l|l|l|l|l|l|l|l|l|l|l|l|l|l|l|l|l|l|l|l|l|l|l|l}
\hline 11 & 0 & 0 & 0 & 0 & 0 & 0 & 0 & 0 & 0 & 0 & 0 & 0 & 4 & 5 & 4 & 4 & 5 & 4 & 2 & 4 & 2 & 2 & 4 & 5 & 2 \\
\hline 12 & 0 & 0 & 0 & 0 & 0 & 0 & 0 & 0 & 0 & 0 & 0 & 0 & 0 & 4 & 3 & 2 & 4 & 1 & 0 & 2 & 0 & 5 & 3 & 0 & 0 \\
\hline 13 & 0 & 0 & 0 & 0 & 0 & 0 & 0 & 0 & 0 & 0 & 0 & 0 & 0 & 0 & 4 & 1 & 3 & 3 & 5 & 4 & 3 & 4 & 4 & 0 & 2 \\
\hline 14 & 0 & 0 & 0 & 0 & 0 & 0 & 0 & 0 & 0 & 0 & 0 & 0 & 0 & 0 & 0 & 2 & 2 & 0 & 2 & 5 & 3 & 5 & 4 & 1 & 3 \\
\hline 15 & 0 & 0 & 0 & 0 & 0 & 0 & 0 & 0 & 0 & 0 & 0 & 0 & 0 & 0 & 0 & 0 & 0 & 1 & 3 & 3 & 4 & 3 & 0 & 2 & 3 \\
\hline 16 & 0 & 0 & 0 & 0 & 0 & 0 & 0 & 0 & 0 & 0 & 0 & 0 & 0 & 0 & 0 & 0 & 0 & 2 & 1 & 0 & 4 & 0 & 3 & 1 & 4 \\
\hline 17 & 0 & 0 & 0 & 0 & 0 & 0 & 0 & 0 & 0 & 0 & 0 & 0 & 0 & 0 & 0 & 0 & 0 & 0 & 0 & 2 & 5 & 1 & 3 & 1 & 0 \\
\hline 18 & 0 & 0 & 0 & 0 & 0 & 0 & 0 & 0 & 0 & 0 & 0 & 0 & 0 & 0 & 0 & 0 & 0 & 0 & 0 & 0 & 0 & 1 & 0 & 5 & 0 \\
\hline 19 & 0 & 0 & 0 & 0 & 0 & 0 & 0 & 0 & 0 & 0 & 0 & 0 & 0 & 0 & 0 & 0 & 0 & 0 & 0 & 0 & 0 & 4 & 2 & 5 & 2 \\
\hline 20 & 0 & 0 & 0 & 0 & 0 & 0 & 0 & 0 & 0 & 0 & 0 & 0 & 0 & 0 & 0 & 0 & 0 & 0 & 0 & 0 & 0 & 3 & 5 & 0 & 3 \\
\hline 21 & 0 & 0 & 0 & 0 & 0 & 0 & 0 & 0 & 0 & 0 & 0 & 0 & 0 & 0 & 0 & 0 & 0 & 0 & 0 & 0 & 0 & 0 & 3 & 1 & 0 \\
\hline 22 & 0 & 0 & 0 & 0 & 0 & 0 & 0 & 0 & 0 & 0 & 0 & 0 & 0 & 0 & 0 & 0 & 0 & 0 & 0 & 0 & 0 & 0 & 0 & 2 & 2 \\
\hline 23 & 0 & 0 & 0 & 0 & 0 & 0 & 0 & 0 & 0 & 0 & 0 & 0 & 0 & 0 & 0 & 0 & 0 & 0 & 0 & 0 & 0 & 0 & 0 & 0 & 5 \\
\hline 24 & 0 & 0 & 0 & 0 & 0 & 0 & 0 & 0 & 0 & 0 & 0 & 0 & 0 & 0 & 0 & 0 & 0 & 0 & 0 & 0 & 0 & 0 & 0 & 0 & 0 \\
\hline
\end{tabular}

Table 3. Penalty cost of passing through the forbidden zones between nodes

\begin{tabular}{|c|c|c|c|c|c|c|c|c|c|c|c|c|c|c|c|c|c|c|c|c|c|c|c|c|c|}
\hline & & 1 & 2 & 3 & 4 & 5 & 6 & 7 & 8 & 9 & 10 & 11 & 12 & 13 & 14 & 15 & 16 & 17 & 18 & 19 & 20 & 21 & 22 & 23 & 24 \\
\hline 0 & 0 & 275 & 0 & 247 & 0 & 274 & 0 & 0 & 240 & 0 & 2071 & 2013 & 0 & 2094 & 0 & 2666 & 0 & 0 & 0 & 2842 & 0 & \begin{tabular}{|l|}
2578 \\
\end{tabular} & \begin{tabular}{|l|}
1513 \\
\end{tabular} & 1357 & 1442 \\
\hline 1 & ( & 0 & 239 & 2 & 0 & 283 & 256 & 207 & 0 & 0 & 2652 & 1104 & 0 & 1076 & 0 & 0 & 0 & 2154 & 1596 & 0 & 1864 & 2643 & 0 & 983 & 782 \\
\hline 2 & c & 0 & 0 & 51 & \begin{tabular}{|l|l|}
114 \\
\end{tabular} & 0 & 273 & 0 & \begin{tabular}{|l|l|}
172 \\
\end{tabular} & 254 & 0 & 2509 & 2992 & 0 & 0 & 0 & 0 & 1873 & 1268 & 1404 & 0 & 0 & \begin{tabular}{|l|}
1883 \\
\end{tabular} & 0 & 156 \\
\hline 3 & 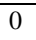 & 0 & $\overline{0}$ & 0 & 0 & 105 & 0 & 0 & 0 & 190 & 2735 & 1721 & 2438 & 0 & 0 & 0 & 0 & \begin{tabular}{|l|}
1657 \\
\end{tabular} & 0 & 2306 & 0 & 0 & 2603 & & 0 \\
\hline 4 & c & 0 & 0 & 0 & 0 & 281 & 0 & 188 & 0 & 0 & 0 & 0 & 2920 & 0 & 0 & 1579 & 2761 & 0 & 2379 & 2954 & 1666 & 0 & 0 & 2709 & 0 \\
\hline 5 & ( & 0 & 0 & 0 & 0 & 0 & 231 & 290 & 200 & 193 & 1808 & 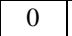 & 0 & 0 & 2116 & 0 & 2741 & 1246 & 0 & 1188 & 1045 & 0 & 0 & 0 & 0 \\
\hline 6 & 0 & 0 & 0 & 0 & 0 & 0 & 0 & 0 & 222 & 0 & 0 & 0 & 0 & 0 & \begin{tabular}{|l|}
2865 \\
\end{tabular} & 0 & 0 & $\mid 1142$ & 0 & 0 & 0 & 1636 & 1970 & 2749 & 260 \\
\hline 7 & 0 & 0 & 0 & 0 & 0 & 0 & 0 & 0 & 237 & 0 & 0 & 0 & 0 & 2946 & 0 & 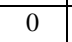 & 0 & 0 & \begin{tabular}{|l|}
1662 \\
\end{tabular} & 0 & 2395 & 0 & 0 & 0 & 0 \\
\hline 8 & 0 & 0 & 0 & 0 & 0 & 0 & 0 & 0 & 0 & 0 & 0 & 1270 & 2756 & 1169 & 2140 & 0 & 1429 & 1128 & 1225 & 0 & 2797 & 2246 & 0 & 0 & 223 \\
\hline 9 & 0 & 0 & 0 & 0 & 0 & 0 & 0 & 0 & 0 & 0 & 0 & 0 & 0 & 0 & \begin{tabular}{|l|}
1033 \\
\end{tabular} & 2649 & 1549 & 0 & 0 & 1103 & 0 & 2009 & 0 & 0 & 0 \\
\hline 10 & 0 & 0 & 0 & 0 & 0 & 0 & 0 & 0 & 0 & 0 & & 0 & 0 & & 0 & 0 & 1052 & 1440 & 2491 & 0 & 0 & 1185 & $\mid 1237$ & 0 & 0 \\
\hline 11 & 0 & 0 & 0 & 0 & 0 & 0 & 0 & 0 & 0 & 0 & 0 & 0 & 0 & 2725 & 0 & 1385 & 1174 & 0 & 0 & 0 & 0 & \begin{tabular}{|l|}
1602 \\
\end{tabular} & \begin{tabular}{|l|}
2573 \\
\end{tabular} & 0 & $144 \mathrm{C}$ \\
\hline 12 & 0 & 0 & 0 & 0 & 0 & 0 & 0 & 0 & 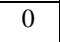 & 0 & & 0 & 0 & 2841 & 2789 & 1451 & 2615 & 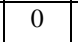 & 0 & & 0 & 2643 & 1980 & 2963 & 117 \\
\hline 13 & 0 & 0 & 0 & 0 & 0 & 0 & 0 & 0 & 0 & 0 & 0 & 0 & 0 & 0 & 2235 & 1020 & 0 & 0 & \begin{tabular}{|l|}
1020 \\
\end{tabular} & 0 & 0 & 0 & 0 & 1679 & 2365 \\
\hline 14 & 0 & 0 & 0 & 0 & 0 & 0 & 0 & 0 & 0 & 0 & c & 0 & & & 0 & 1009 & 2563 & 0 & 2449 & 0 & 0 & 0 & 1623 & 0 & 0 \\
\hline 15 & 0 & 0 & 0 & 0 & 0 & 0 & 0 & 0 & 0 & 0 & 0 & 0 & 0 & 0 & 0 & 0 & 2187 & 0 & \begin{tabular}{|l|}
2007 \\
\end{tabular} & 2864 & 0 & \begin{tabular}{|l|}
1287 \\
\end{tabular} & \begin{tabular}{|l|}
1167 \\
\end{tabular} & 0 & 0 \\
\hline 16 & 0 & 0 & 0 & $\sigma$ & 0 & 0 & 0 & 0 & 0 & 0 & 0 & 0 & & 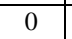 & 0 & 0 & 0 & 1896 & 1976 & 0 & 0 & 0 & 2415 & 2282 & 216 \\
\hline 17 & 0 & 0 & 0 & 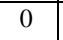 & 0 & 0 & 0 & 0 & 0 & 0 & 0 & 0 & 0 & 0 & 0 & 0 & 0 & 0 & 0 & 0 & 0 & 2927 & \begin{tabular}{|l|}
2585 \\
\end{tabular} & 2002 & 115 \\
\hline 18 & 0 & 0 & 0 & 0 & 0 & 0 & 0 & 0 & 0 & 0 & 0 & 0 & 0 & 0 & 0 & c & 0 & 0 & 0 & 1954 & 0 & 0 & 2251 & 0 & 0 \\
\hline 19 & $\overline{0}$ & 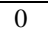 & 0 & , & 0 & 0 & 0 & 0 & 0 & 0 & 0 & 0 & 0 & 0 & $\overline{0}$ & 0 & 0 & 0 & 0 & 0 & 1734 & 2972 & 0 & 0 & 0 \\
\hline 20 & 0 & 0 & 0 & 0 & 0 & 0 & 0 & 0 & 0 & 0 & 0 & 0 & 0 & 0 & 0 & 0 & 0 & 0 & 0 & 0 & 0 & 0 & 2774 & 0 & 0 \\
\hline 21 & 0 & 0 & 0 & 0 & 0 & S & 0 & 0 & 0 & 0 & 0 & 0 & 0 & 0 & 0 & 0 & 0 & 0 & 0 & 0 & 0 & 0 & 0 & 1052 & 0 \\
\hline 22 & 0 & 0 & 0 & 0 & 0 & 0 & 0 & 0 & 0 & 0 & 0 & 0 & 0 & 0 & 0 & 0 & 0 & 0 & 0 & 0 & 0 & 0 & 0 & 2507 & 0 \\
\hline 23 & 0 & 0 & 0 & 0 & 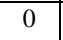 & 0 & 0 & 0 & 0 & 0 & 0 & 0 & 0 & 0 & 0 & 0 & 0 & 0 & U & 0 & 0 & 0 & 0 & 0 & 0 \\
\hline 24 & 0 & 0 & 0 & 0 & 0 & 0 & 0 & 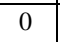 & 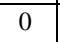 & 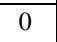 & 0 & 0 & & 0 & 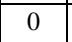 & 0 & 0 & 0 & 0 & 0 & 0 & 0 & 0 & 0 & 0 \\
\hline
\end{tabular}

\section{Results analysis}

The relevant parameters of genetic algorithm are set as follows: the maximum iterative algebra max gen $=700$, population size popsize $=100$, crossover probability $p c=0.9$, mutation probability $p m=0.1, \omega_{2}=0.6$ and $\omega_{3}=0.4$. The following conclusions are made through the programming of genetic algorithm on Matlab R2010b platform and operating it on Intel Core i5 $2.25 \mathrm{GHz}, 2 \mathrm{~Gb}$ RAM for 51.36 seconds: the final distribution map is shown in 3-3, the algorithm convergence is shown in figure 2, and the route and its distribution scheme are shown in table 4. It is known by calculation that the total distribution cost is RMB 139,240.68 and 52 densely populated areas are went through.

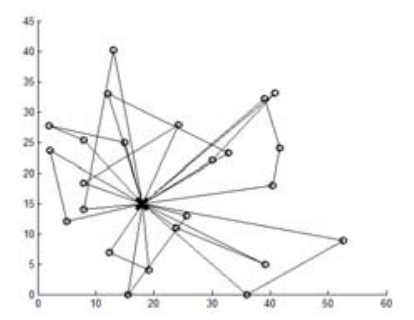

Table 4 Distribution scheme

Fig 2. Distribution map

\begin{tabular}{c|c|c|c}
\hline $\begin{array}{c}\text { Serial } \\
\text { No. }\end{array}$ & $\begin{array}{c}\text { Distribution } \\
\text { route }\end{array}$ & $\begin{array}{c}\text { Serial } \\
\text { No. }\end{array}$ & $\begin{array}{c}\text { Distribution } \\
\text { route }\end{array}$ \\
\hline 1 & $0-13-4-0$ & 7 & $0-17-24-0$ \\
\hline 2 & $0-10-19-12-0$ & 8 & $0-16-15-0$ \\
\hline 3 & $0-22-11-9-0$ & 9 & $0-2-23-0$ \\
\hline 4 & $0-21-7-0$ & 10 & $0-18-17-14-0$ \\
\hline 5 & $0-14-1-8-0$ & 11 & $0-6-10-0$ \\
\hline
\end{tabular}




\section{6}

$0-5-3-20-0$

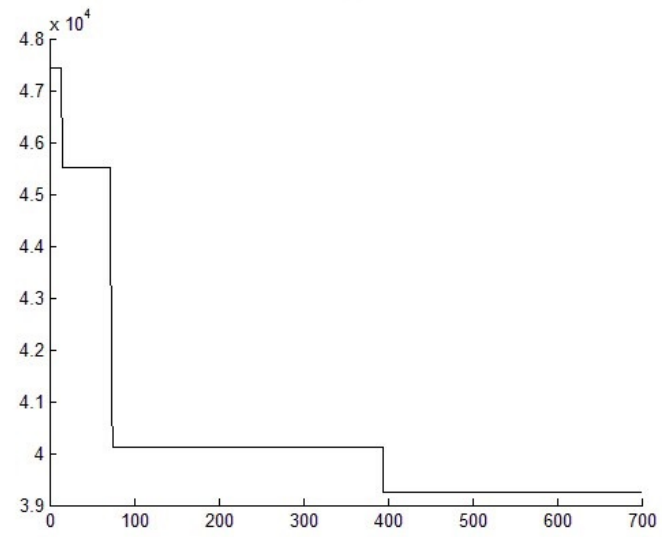

a) Convergence diagram of objective function value 2

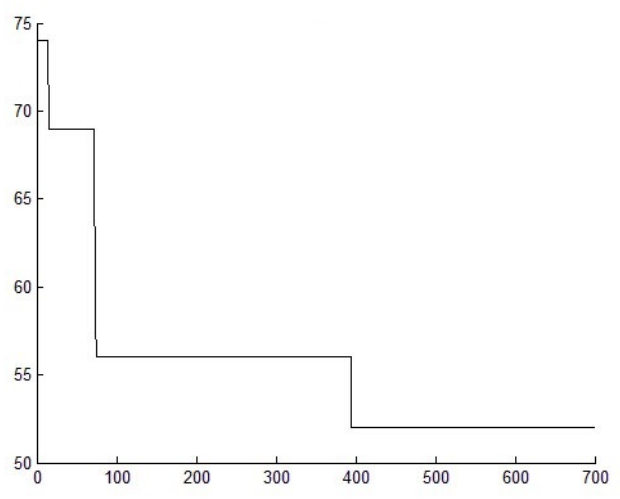

b) Convergence diagram of objective function value 2

Fig 3. Algorithm convergence diagram

\section{Conclusion}

This paper puts forwards the problem concerning the vehicle scheduling optimization of dangerous goods distribution under single distribution center based on security and describes and defines this problem. Based on this, relevant assumptions of the problem are made for the convenience of building the mathematical model. Finally, the model is solved by designing a multi-target genetic algorithm based on weight and the reliability and validity of the model are verified by designing the related examples. The research results show that the model has high reliability and good solving ability and can obtain a satisfied solution of vehicle scheduling optimization problem of dangerous goods distribution under single distribution center in a relatively short time.

\section{References}

[1] Jiang Lili. Research on Development of Ningbo Bonded Logistics Center (B)[J]. Journal of Ningbo University of Engineering, 2007, 19(3): 31-34.

[2] Qu Xianfeng, Zuo Chunrong. An Improved Genetic Algorithm and Its Application in VRP [J]. [3] Science Technology and Engineering, 2008, 8(2):563-565.

[3] Jiang Dali, Yang Xilong, Du Wen. Research on Genetic Algorithm of Vehicle Route Problem[J]. System Engineering Theory and Practice, 1996, 6:40-45.

[4] Zhou Kun. Research on Vehicle Scheduling Optimization Problem of Dangerous Goods Transportation under Single Distribution Center based on Security[D]. Southwest Jiaotong University, 2012. 